\section{OTU-030 FAECAL MICROBIOTA TRANSPLANTATION FOR RECURRENT CLOSTRIDIUM DIFFICILE INFECTION: REAL WORLD UK DATA}

${ }^{1} J o n a t h a n$ Digby-Bell*, ${ }^{2}$ Kamal Patel, ${ }^{3}$ Mohamed Mutalib, ${ }^{1}$ Peter Inving, ${ }^{1}$ Simon Goldenberg. 'Guy's and St Thomas' NHS Trust, London, UK; ${ }^{2}$ St George's Hospital, London, UK; ${ }^{3}$ Evelina Children's Hospital, London, UK

\subsection{6/gutjnl-2018-BSGAbstracts.444}

Introduction recurrent Clostridium difficile infection (rCDI) is a medically challenging condition with limited therapeutic options often resulting in repeated admissions to hospital with associated high financial and patient burden. Faecal microbiota transplantation (FMT) has been shown to be highly effective treatment in placebo-controlled trials, however little real life data exists particularly from UK centres. We report our experience in a single tertiary referral centre.

Method Data was collected prospectively from January 2015 to November 2017 for patient demographics, comorbidities, route of FMT administration, 30 day and 1 year mortality. Primary endpoint was resolution of diarrhoea without relapse 10 weeks after first FMT. Resolution of diarrhoea without relapse 10 weeks after second FMT was also recorded.

Results 35 adult and 2 paediatric patients were approved for FMT for treatment of rCDI of which 35 patients underwent the procedure. 2 patients clinically deteriorated before FMT could be performed such that FMT became inappropriate.

$27 / 35(77 \%)$ were female, with a mean age of 67 y (range 4-91), and a mean ASA grade of 2.0 (0-4). Patients had received $3.1(2-5)$ courses of antibiotics for clostridium difficile and $27(77 \%)$ were external referrals. 32 FMTs were performed via colonoscopy and 3 via nasojejunal tube.

3 patients died within 30 days of FMT (mean ASA grade 3.3) but none directly related to the FMT or C Diff. There was once further expected death 90 days after FMT. No other major side effects or safety concerns were seen.

Of the patients who survived to day $30 ; 28$ out of 32 $(87.5 \%)$ patients had cessation of diarrhoea without relapse after 10 weeks. 4 patients had recurrent diarrhoea within 10 weeks of FMT of whom 2 had a 2 nd FMT resulting in cessation of diarrhoea with no relapse after 10 weeks. The other two patients clinically deteriorated due to underlying medical conditions such that a 2nd FMT was inappropriate. All patients who survived had resolution of symptoms after their first or second transplant.

Conclusion FMT is a highly effective treatment for rCDI in the real world with resolution of symptoms and no relapse after 10 weeks achieved in $89.7 \%$ of patients undergoing 1 st FMT increasing to $100 \%$ after a 2 nd FMT.

No safety concerns were identified during the study period. The 3 deaths within 30 days of FMT highlight the comorbid population who develop rCDI and better patient selection is required to ensure appropriateness of FMT in high risk groups.

\section{OTU-031 MY-IBD PORTAL: PROGRESS AND IMPACT UPDATE}

\footnotetext{
1,2John Mclaughlin*, 'Sister Catherine Stansfield, 'Simon Lal, ${ }^{3}$ Keith Bodger, 'James Andrew Robinson. 'Salford Royal Hospitals, Salford, UK; ${ }^{2}$ University of Manchester, Manchester, UK; ${ }^{3}$ University of Liverpool, Liverpool, UK
}

\subsection{6/gutjnl-2018-BSGAbstracts.445}

Introduction Patients with Inflammatory Bowel Disease (IBD) often struggle with access to prompt advice regarding and place a heavy reliance on overstretched services. We have a track record in guided self-management of IBD so explored whether this could be built into a novel patient-centred portal with direct on line access to a fully personalised health record, integrating individualised plans of care and disease monitoring tools.

Methods In a unique collaboration with local patients, the patient charity Crohn's and Colitis UK, health care and IT teams, we developed, implemented and evaluated a web based portal at Salford Royal. At the outset, a user group of patients was established and their views and needs were central to each step of the development, refinement and evaluation of the portal. From this group it was clear that there was a particular demand by IBD patients to have access to their health records and reliable information.

Results We have built and refined the 'MY IBD' portal, which is fully integrated with the electronic patient record and delivers:

1. Access to personalised information: diagnosis, visual aids and links to CCUK information resources

2. Immediate access to blood results, investigation results, clinic letters

3. Disease activity assessment tools

4. A personalised plan of care available online for the patient

5. Improved communication with the IBD team: messaging facility and trigger emails when disease activity scores are high.

720 patients are now using the portal. Overall usability was scored as excellent, showing patients were helped with decision-making. Improvements were seen in perceived support $(\mathrm{p}=0.06)$ compared to non-users with a trend to improved disease related knowledge $(p=0.14)$. An average of clinic 2.9 attendances per year (2014-15) reduced to 0.6 attendances for portal self-management users (2016-2017) releasing over 500 clinic appointments. Satisfaction with self-management remains high with $98 \%$ of patients rating the process as good/ excellent. User group sessions have captured value to patients. They expressed it had 'completely changed my care', 'having up to date information about your condition leads to less stress and better health' and 'puts patients back in control of their illness'.

The portal has also been refined for integration with the IBD registry.

Conclusions Patient experience and service have improved. Portal patients are more in control, with greater independence to self-manage through better understanding of their condition. Other Trusts are now seeking to adopt the portal and we welcome enquiries to establish it in other NHS sites.

Acknowledgments funded by CCUK, 'Living with IBD'.

\section{OTU-032 INTEGRATED IBS CARE PATHWAY WITH DIRECT- ACCESS, DIETETIC-LED SERVICE - IMPACT ON PATIENT INVESTIGATION AND OUTCOMES}

Claire Oldale*, Phoebe Hodges, Samuel Waternan, Alexandra Di Mambro. Glos Hospitals NHS Foundation Trust, Cheltenham, UK

10.1136/gutjnl-2018-BSGAbstracts.446

Introduction An integrated care pathway with direct access to a dietitian-led refractory IBS (RIBS) service was set up in Gloucestershire in 2016. GPs may refer patients $<45$ years with symptoms fulfilling ROME criteria for IBS which is refractory to first 